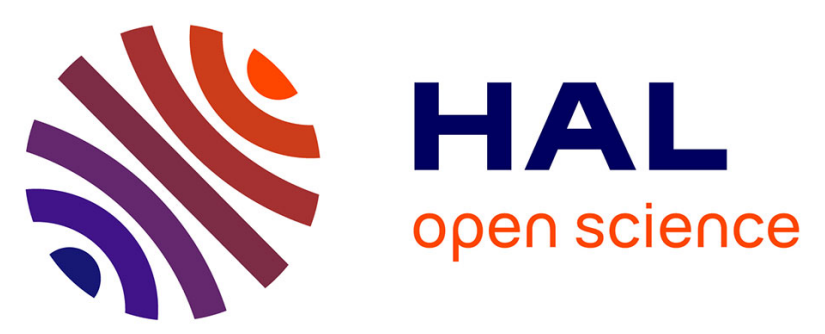

\title{
A Novel Approach to Necessary and Sufficient Conditions for the Diagonalization of Refined Neutrosophic Matrices
}

Necati Olgun, Ahmed Hatip, Mikail Bal, Mohammad Abobala

\section{- To cite this version:}

Necati Olgun, Ahmed Hatip, Mikail Bal, Mohammad Abobala. A Novel Approach to Necessary and Sufficient Conditions for the Diagonalization of Refined Neutrosophic Matrices. International Journal of Neutrosophic Science, 2021, pp.72 - 79. 10.54216/ijns.160202 . hal-03428932

\section{HAL Id: hal-03428932 \\ https://hal.science/hal-03428932}

Submitted on 5 Dec 2021

HAL is a multi-disciplinary open access archive for the deposit and dissemination of scientific research documents, whether they are published or not. The documents may come from teaching and research institutions in France or abroad, or from public or private research centers.
L'archive ouverte pluridisciplinaire HAL, est destinée au dépôt et à la diffusion de documents scientifiques de niveau recherche, publiés ou non, émanant des établissements d'enseignement et de recherche français ou étrangers, des laboratoires publics ou privés. 


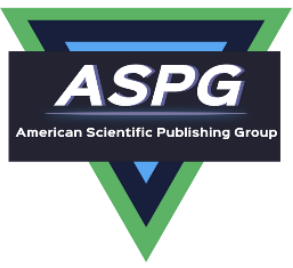

\title{
A Novel Approach to Necessary and Sufficient Conditions for the Diagonalization of Refined Neutrosophic Matrices
}

\author{
Necati Olgun ${ }^{1}$, Ahmed Hatip ${ }^{2}$, Mikail Bal ${ }^{3}$, Mohammad Abobala ${ }^{4}$ \\ ${ }^{1}$ Department of Mathematics, Gaziantep University, Gaziantep27310, Turkey \\ ${ }^{2}$ Department of Mathematics, Gaziantep University, Turkey \\ ${ }^{3}$ Department of Mathematics, Gaziantep University, Gaziantep 27310, Turkey \\ ${ }^{4}$ Tishreen University, Faculty of Science, Department of Mathematics, Lattakia, Syria \\ Emails: olgun@gantep.edu.tr kollnaar5@gmail.com, ; mikailbal46@hotmail.com, \\ Mohammadabobala777@gmail.com
}

\begin{abstract}
This work is dedicated to study the conditions of diagonalization in the case of refined neutrosophic matrices, where it presents the necessary and sufficient conditions for the diagonalization of these matrices by finding a relationship with classical diagonalization of matrices. Also, it describes an algorithm to obtain all eigen values and eigen vectors of refined neutrosophic matrices from the classical ones.
\end{abstract}

Keywords: Refined neutrosophic matrix, refined neutrosophic eigen value, refined neutrosophic eigen vector, refined neutrosophic diagonalization

\section{Introduction}

Neutrosophy is a new branch of philosophy founded by F. Smarandache [16] to deal with uncertainty in all fields of human knowledge, where it can be considered as a generalization of intuitionistic fuzzy logic.

Neutrosophic algebraic studies began with the great efforts of Kandasamy and Smarandache in [12], where they studied for the first time neutrosophic rings and neutrosophic groups.

In the literature, we find many neutrosophic algebraic results about spaces [11], modules [6], rings [5,13], number theory [15,17], and other related systems [23-25] 
Neutrosophic matrices were defined in [9] as a useful tool to deal with indeterminacy and as a generalization of fuzzy matrices [10]. The algebraic properties of these matrices were studied in [8], such as their linear transformations and diagonalization problem [27].

In [7], Agboola et. al. presented the idea of splitting the indeterminacy element I into two levels of indeterminacy $I_{1}, I_{2}$ with the following property $I_{1} I_{2}=I_{2} I_{1}=I_{1}, I_{1}{ }^{2}=I_{1}, I_{2}{ }^{2}=I_{2}$. This idea was used to defined refined neutrosophic groups [7], refined neutrosophic rings [1], modules [14,18], and matrices [4].

The invertibility, nilpotency, and idempotency of refined neutrosophic matrices were characterized in [4].

In this work, we study the problem of diagonalization of refined neutrosophic matrices, where we determine an algorithm to find all eigen vectors and values, and we use this idea to determine the necessary and sufficient condition for the diagonalization of refined neutrosophic matrices.

All refined neutrosophic matrices through this study are considered over a refined neutrosophic field $K\left(I_{1}, I_{2}\right)$.

\section{Preliminaries}

Definition: [7]

If $X$ is a set then $X\left(I_{1}, I_{2}\right)=\left\{\left(a, b I_{1}, c I_{2}\right) ; a, b, c \in X\right\}$ is called the refined neutrosophic generated by $X, I_{1}, I_{2}$.

Definition: [1]

Let $(R,+,$.$) be a ring then \left(R\left(I_{1}, I_{2}\right),+,.\right)$ is called a refined neutrosophic ring generated by $R, I_{1}, I_{2}$.

Where $I_{1}^{2}=I_{1}, I_{2}^{2}=I_{2}, I_{1} I_{2}=I_{2} I_{1}=I_{1}$.

If $\mathrm{R}$ is an algebraic field, then $R\left(I_{1}, I_{2}\right)$ is called a refined neutrosophic field.

Definition: [4]

Let $A=\left[\begin{array}{ccc}a_{11} & \ldots . & a_{1 m} \\ \vdots & \ddots & \vdots \\ a_{n 1} & \ldots & a_{n m}\end{array}\right]$ be an $n \times m$ mat اكتب المعادلة .rix, if $a_{i j}=x+y I_{1}+z I_{2} \in R_{2}(I)$, then it is called an refined neutrosophic matrix. Where $R_{2}(I)$ is an refined neutrosophic field.

Theorem: [4]

Let $X=A+B I_{1}+C I_{2}$ be a square $n \times n$ refined neutrosophic matrix, then it is invertible if and only if $A, A+C, A+B+C$ are invertible. The inverse of $X$ is

$$
X^{-1}=A^{-1}+\left((A+B+C)^{-1}-(A+C)^{-1}\right) I_{1}+\left((A+C)^{-1}-A^{-1}\right) I_{2} .
$$

Definition: [4]

We defined the determinant of a square $n \times n$ refined neutrosophic matrix as

$$
\operatorname{det} X=\operatorname{det} A+[\operatorname{det}(A+B+C)-\operatorname{det}(A+C)] I_{1}+[\operatorname{det}(A+C)-\operatorname{det} A] I_{2} .
$$




\section{Main results}

\section{Definition 3.1:}

Let $L=A+B I_{1}+C I_{2}$ be a refined neutrosophic matrix, and $M=X+Y I_{1}+Z I_{2}$ is a strong refined neutrosophic vector, then it is called a refined neutrosophic eigen vector of $L$ if and only if

$L M=\left(a+b I_{1}+c I_{2}\right) M$.

The refined neutrosophic number $a+b I_{1}+c I_{2}$ is called a refined neutrosophic eigen value.

\section{Theorem 3.2:}

Let $L=A+B I_{1}+C I_{2}$ be a refined neutrosophic matrix, then $M=X+Y I_{1}+Z I_{2}$ is a refined neutrosophic eigen vector with $a+b I_{1}+c I_{2}$ as the corresponding eigen value if and only if $X, X+Z, X+Y+Z$ are eigen vectors of $A, A+C, A+B+C$ respectively. As well as, $a, a+c, a+b+c$ are the corresponding eigen values respectively.

\section{Proof:}

Suppose that $M=X+Y I_{1}+Z I_{2}$ is a refined neutrosophic eigen vector with $a+b I_{1}+c I_{2}$ as the corresponding eigen value, then $L M=\left(a+b I_{1}+c I_{2}\right) M$. By easy computing, we get

$\left(A X+I_{1}[(A+B+C)(X+Y+Z)-(A+C)(X+Z)]+I_{2}[(A+C)(X+Z)-A X]=\right.$

$a X+I_{1}[(a+b+c)(X+Y+Z)-(a+c)(X+Z)]+I_{2}[(a+c)(X+Z)-a X]$. Which is equivalent to

$A X=a X,(A+C)(X+Z)=(a+c)(X+Z),(A+B+C)(X+Y+Z)=(a+b+c)(X+Y+Z)$. Thus

$X, X+Z, X+Y+Z$ are eigen vectors of $A, A+C, A+B+C$ respectively. As well as, $a, a+c, a+b+c$ are the corresponding eigen values respectively.

Conversely, we assume that $X, X+Z, X+Y+Z$ are eigen vectors of $A, A+C, A+B+C$ respectively, with $a$, $a+$ $c, a+b+c$ are the corresponding eigen values respectively, then by the definition of eigen vectors we can write:

$A X=a X,(A+C)(X+Z)=(a+c)(X+Z),(A+B+C)(X+Y+Z)=(a+b+c)(X+Y+Z)$.

This means that $\left(A X+I_{1}[(A+B+C)(X+Y+Z)-(A+C)(X+Z)]+I_{2}[(A+C)(X+Z)-A X]=\right.$ $a X+I_{1}[(a+b+c)(X+Y+Z)-(a+c)(X+Z)]+I_{2}[(a+c)(X+Z)-a X]$. Which implies

$L M=\left(a+b I_{1}+c I_{2}\right) M$, thus $M=X+Y I_{1}+Z I_{2}$ is a refined neutrosophic eigen vector with $a+b I_{1}+c I_{2}$ as the corresponding eigen value.

\section{Theorem 3.3:}

Eigen values can be gotten by solving the refined neutrosophic equation $\operatorname{det}\left(L-\left(a+b I_{1}+c I_{2}\right) U_{n \times n}\right)=0$.

\section{Proof:}

Firstly, we have:

DOI: https://doi.org/10.54216/IJNS.160202

Received: July 15, 2021 Accepted: November 09, 2021 


$$
\begin{aligned}
& \operatorname{det}\left(L-\left(a+b I_{1}+c I_{2}\right) U_{n \times n}\right)=\operatorname{det}\left(\left[A-a U_{n \times n}\right]+\left[B-b U_{n \times n}\right] I_{1}+\left[C-c U_{n \times n}\right] I_{2}\right) \\
& =\operatorname{det}\left(A-a U_{n \times n}\right)+I_{1}\left[\operatorname{det}\left(A+B+C-(a+b+c) U_{n \times n}\right)-\operatorname{det}\left(A+C-(a+c) U_{n \times n}\right)\right]+I_{2}[\operatorname{det}(A+C- \\
& \left.\left.(a+c) U_{n \times n}\right)-\operatorname{det}\left(A-a U_{n \times n}\right)\right]=0 \text {. This implies that } \\
& \operatorname{det}\left(A-a U_{n \times n}\right)=\operatorname{det}\left(A+C-(a+c) U_{n \times n}\right)=\operatorname{det}\left(A+B+C-(a+b+c) U_{n \times n}\right)=0 .
\end{aligned}
$$

Hence, $a, a+c, a+b+c$ are eigen values of $A, A+C, A+B+C$ respectively, which is equivalent to that $a+b I_{1}+c I_{2}$ is a refined neutrosophic eigen value of $\mathrm{L}$.

\section{Example 3.4:}

Consider the following refined neutrosophic matrix $L=\left[\begin{array}{cc}1-I_{1}+I_{2} & 1-I_{2} \\ -I_{1}+I_{2} & -1-I_{1}+4 I_{2}\end{array}\right]$.

(a) $\mathrm{L}$ is written as $L=A+B I_{1}+C I_{2}$. Where $A=\left(\begin{array}{cc}1 & 1 \\ 0 & -1\end{array}\right), B=\left(\begin{array}{cc}-1 & 0 \\ -1 & -1\end{array}\right), C=\left(\begin{array}{cc}1 & -1 \\ 1 & 4\end{array}\right)$.

(b) We have $A+C=\left(\begin{array}{ll}2 & 0 \\ 1 & 3\end{array}\right), A+B+C=\left(\begin{array}{ll}1 & 0 \\ 0 & 2\end{array}\right)$. The set of eigen values of $\mathrm{A}$ is $\{1,-1\}$, for $A+C$ it is $\{2,3\}$, and for $A+B+C$ it is $\{1,2\}$.

(c) According to Theorem 3.2, the set of refined neutrosophic eigen values of $\mathrm{L}$ is

$$
\begin{aligned}
\left\{1+I_{1}[1-2]+\right. & I_{2}[2-1], 1+I_{1}[2-2]+I_{2}[2-1], 1+I_{1}[1-3]+I_{2}[3-1], 1+I_{1}[2-3]+I_{2}[3-1],-1 \\
& +I_{1}[1-2]+I_{2}[2+1],-1+I_{1}[2-2]+I_{2}[2+1],-1+I_{1}[1-3]+I_{2}[3+1],-1 \\
& \left.+I_{1}[2-3]+I_{2}[3+1]\right\} \\
=\left\{1-I_{1}+I_{2}, 1+\right. & \left.I_{2}, 1-2 I_{1}+2 I_{2}, 1-I_{1}+2 I_{2},-1-I_{1}+3 I_{2},-1+3 I_{2},-1-2 I_{1}+4 I_{2},-1-I_{1}+4 I_{2}\right\} .
\end{aligned}
$$

(d) We get the same values by solving the following refined neutrosophic equation:

$\operatorname{det}\left(L-\left(a+b I_{1}+c I_{2}\right) U_{n \times n}\right)=\left(a+b I_{1}+c I_{2}\right)^{2}+\left(a+b I_{1}+c I_{2}\right)\left(2 I_{1}-5 I_{2}\right)+\left(-1-4 I_{1}+7 I_{2}\right)=0$. For the solution, we can use the algebraic algorithm which was introduced in [3].

\section{Definition 3.5:}

Let $X=A+B I_{1}+C I_{2}$ be any refined neutrosophic matrix, then it is called diagonalizable if there exists an invertible refined neutrosophic matrix $Y=F+G I_{1}+H I_{2}$ such that $Y^{-1} X Y=D$. Where D is a refined neutrosophic diagonal matrix.

\section{Theorem 3.6:}

Let $X=A+B I_{1}+C I_{2}$ be any refined neutrosophic matrix, then it is diagonalizable if and only if $A, A+C, A+B+$ $C$ are diagonalizable.

\section{Proof:}

Suppose that $A, A+C, A+B+C$ are diagonalizable, then there are three invertible matrices $\mathrm{F}, \mathrm{G}, \mathrm{H}$ such that $F^{-1} A F=D_{0}, G^{-1}(A+B+C) G=D_{1}, H^{-1}(A+C) H=D_{2}$. Where $D_{0}, D_{1}, D_{2}$ are diagonal matrices. Put $Y=F+$ $(G-H) I_{1}+(H-F) I_{2}, D=D_{0}+\left(D_{1}-D_{2}\right) I_{1}+\left(D_{2}-D_{0}\right) I_{2}$. Now, let us compute $Y^{-1} X Y=\left(F^{-1}+I_{1}[(G-\right.$ DOI: https://doi.org/10.54216/IJNS.160202

Received: July 15, 2021 Accepted: November 09, 2021 
$\left.\left.H+H-F+F)^{-1}-(H-F+F)^{-1}\right]+I_{2}\left[(H-F+F)^{-1}-F^{-1}\right]\right)\left(A+B I_{1}+C I_{2}\right)\left(F+(G-H) I_{1}+(H-F)\right)$

$=$

$\left(F^{-1}+I_{1}\left[G^{-1}-H^{-1}\right]+I_{2}\left[H^{-1}-F^{-1}\right]\right)\left(A F+I_{1}[(A+B+C)(G)-(A+C) H]+I_{2}[(A+C) H-A F]\right)=$ $F^{-1} A F+I_{1}\left[G^{-1}(A+B+C) G-H^{-1}(A+C) H\right]+I_{2}\left[H^{-1}(A+C) H-F^{-1} A F\right]=D_{0}+\left(D_{1}-D_{2}\right) I_{1}+\left(D_{2}-\right.$ $\left.D_{0}\right) I_{2}=D$. This means that $\mathrm{X}$ is diagonalizable.

Conversely, we assume that $\mathrm{X}$ is diagonalizable, hence there exists an invertible refined neutrosophic matrix $Y=$ $F+G I_{1}+H I_{2}$ and a refined diagonal neutrosophic matrix $D=D_{0}+D_{1} I_{1}+D_{2} I_{2}$ such that $Y^{-1} X Y=D(*)$.

We shall compute equation $(*)$ :

$\left(F^{-1}+I_{1}\left[(F+G+H)^{-1}-(F+H)^{-1}\right]+I_{2}\left[(F+H)^{-1}-F^{-1}\right]\right)\left(A+I_{1}[B]+I_{2}[C]\right)\left(F+I_{1}[G]+I_{2}[H]\right)=$ $\left(F^{-1}+I_{1}\left[(F+G+H)^{-1}-(F+H)^{-1}\right]+I_{2}\left[(F+H)^{-1}-F^{-1}\right]\right)\left(A F+I_{1}[(A+B+C)(F+G+H)-(A+\right.$ C) $\left.(F+H)]+I_{2}[(A+C)(F+H)-A F]\right)=$

$F^{-1} A F+I_{1}\left[(F+G+H)^{-1}(A+B+C)(F+G+H)-(F+H)^{-1}(A+C)(F+H)\right]+I_{2}\left[(F+H)^{-1}(A+\right.$ C) $\left.(F+H)-F^{-1} A F\right]=D_{0}+D_{1} I_{1}+D_{2} I_{2}$.

This implies that $F^{-1} A F=D_{0},(F+H)^{-1}(A+C)(F+H)-F^{-1} A F=D_{2},(F+G+H)^{-1}(A+B+C)(F+G+$ $H)-(F+H)^{-1}(A+C)(F+H)=D_{1}$

Thus $(F+H)^{-1}(A+C)(F+H)=D_{0}+D_{2},(F+G+H)^{-1}(A+B+C)(F+G+H)=D_{0}+D_{1}+D_{2}$. Which means that $A, A+C, A+B+C$ are diagonalizable.

\section{Remark 3.7:}

If $F, G, H$ are the diagonalization matrices of $A, A+B+C, A+C$ respectively, then $F+(G-H) I_{1}+(H-F) I_{2}$ is the diagonalization matrix of $X$. Also, the corresponding diagonal matrix of $X$ is

$D=D_{0}+\left(D_{1}-D_{2}\right) I_{1}+\left(D_{2}-D_{0}\right) I_{2}$, where $D_{0}, D_{1}, D_{2}$ are the corresponding diagonal matrices of $A, A+B+$ $C, A+C$ respectively.

\section{Example 3.8:}

Consider the refined neutrosophic matrix defined in the Example 3.4, we have:

(a) The diagonalization matrix of $A$ is $F=\left(\begin{array}{cc}1 & 1 \\ -2 & 0\end{array}\right)$, the corresponding diagonal matrix is $D_{0}=\left(\begin{array}{cc}-1 & 0 \\ 0 & 1\end{array}\right)$. It is clear that $F^{-1} A F=D_{0}$.

(b) The diagonalization matrix of $A+B+C$ is $G=\left(\begin{array}{ll}1 & 0 \\ 0 & 1\end{array}\right)$, the corresponding diagonal matrix is $D_{1}=\left(\begin{array}{ll}1 & 0 \\ 0 & 2\end{array}\right)$. It is clear that $G^{-1}(A+B+C) G=D_{1}$.

(c) The diagonalization matrix of $A+C$ is $H=\left(\begin{array}{cc}1 & 0 \\ -1 & 1\end{array}\right)$, the corresponding diagonal matrix is $D_{2}=\left(\begin{array}{ll}2 & 0 \\ 0 & 3\end{array}\right)$. It is clear that $H^{-1}(A+C) H=D_{2}$.

(d) The refined neutrosophic diagonalization matrix of $\mathrm{L}$ is :

DOI: https://doi.org/10.54216/IJNS.160202

Received: July 15, 2021 Accepted: November 09, 2021 


$$
\begin{aligned}
& Y=F+(G-H) I_{1}+(H-F) I_{2}=\left(\begin{array}{cc}
1 & 1-I_{2} \\
-2+I_{1}+I_{2} & I_{2}
\end{array}\right) . \text { The corresponding diagonal matrix is } \\
& D=D_{0}+\left(D_{1}-D_{2}\right) I_{1}+\left(D_{2}-D_{0}\right) I_{2}=\left(\begin{array}{cc}
-1-I_{1}+3 I_{2} & 0 \\
0 & 1-I_{1}+2 I_{2}
\end{array}\right) . \\
& \text { (e) } F^{-1}=\left(\begin{array}{cc}
0 & -\frac{1}{2} \\
1 & \frac{1}{2}
\end{array}\right), G^{-1}=\left(\begin{array}{ll}
1 & 0 \\
0 & 1
\end{array}\right), H^{-1}=\left(\begin{array}{ll}
1 & 0 \\
1 & 1
\end{array}\right), Y^{-1}=F^{-1}+\left(G^{-1}-H^{-1}\right) I_{1}+\left(H^{-1}-F^{-1}\right) I_{2} \\
& =\left(\begin{array}{cc}
I_{2} & -\frac{1}{2}+\frac{1}{2} I_{2} \\
1-I_{1} & \frac{1}{2}+\frac{1}{2} I_{2}
\end{array}\right), Y^{-1} L Y=D .
\end{aligned}
$$

\section{Conclusion}

In this article, we have studied the necessary and sufficient conditions for the diagonalization of refined neutrosophic matrices. Also, we have determined an algorithm to compute all refined neutrosophic eigen values/vectors which are related to these matrices.

\section{References}

[1] Adeleke, E.O., Agboola, A.A.A., and Smarandache, F., "Refined Neutrosophic Rings II", International Journal of Neutrosophic Science, Vol. 2, pp. 89-94, 2020.

[2] Abobala, M., "On The Characterization of Maximal and Minimal Ideals In Several Neutrosophic Rings", Neutrosophic Sets and Systems, Vol. 45, 2021.

[3] Abobala, M., "On Some Neutrosophic Algebraic Equations", Journal of New Theory, Vol. 33, 2020.

[4] Abobala, M., " On Refined Neutrosophic Matrices and Their Application In Refined Neutrosophic Algebraic Equations", Journal Of Mathematics (Hindawi), 2021.

[5] M. Ali, F. Smarandache, M. Shabir and L. Vladareanu, "Generalization of Neutrosophic Rings and Neutrosophic Fields," Neutrosophic Sets and Systems, vol. 5, pp. 9-14, 2014.

[6] Abobala, M., and Hatip, A., "AH-Substructures In Strong Refined Neutrosophic Modules", International Journal of Neutrosophic Science, Vol. 9, pp. 110-116, 2020.

[7] Agboola, A.A.A., "On Refined Neutrosophic Algebraic Structures", Neutrosophic Sets and Systems, Vol.10, pp. 99-101, 2015.

[8] Abobala, M., On The Representation Of Neutrosophic Matrices By Neutrosophic Linear Transformations", Journal Of Mathematics (Hindawi), 2021.

[9]Dhar M., Broumi S.,and Smarandache F., "A Note on Square Neutrosophic Fuzzy Matrices", Neutrosophic Sets and Systems", Vol. 3, pp. 37-41 2014.

[10] Khaled, H., and Younus, A., and Mohammad, A., " The Rectangle Neutrosophic Fuzzy Matrices", Faculty of Education Journal Vol. 15, 2019. (Arabic version).

DOI: https://doi.org/10.54216/IJNS.160202

Received: July 15, 2021 Accepted: November 09, 2021 
[11] Ibrahim, M.A., Agboola, A.A.A, Badmus, B.S., and Akinleye, S.A., "On refined Neutrosophic Vector Spaces I", International Journal of Neutrosophic Science, Vol. 7, pp. 97-109, 2020.

[12] W. B. V. Kandasamy and F. Smarandache, Neutrosophic Rings, Hexis, Phoenix, Arizona: Infinite Study, 2006.

[13] Sankari, H., and Abobala, M.," AH-Homomorphisms In Neutrosophic Rings and Refined Neutrosophic Rings", Neutrosophic Sets and Systems, Vol. 38, 2020.

[14] Sankari, H., and Abobala, M., "n-Refined Neutrosophic Modules", Neutrosophic Sets and Systems, Vol. 36 , 2020 .

[15] Sankari, H., and Abobala, M.," Neutrosophic Linear Diophantine Equations With two Variables", Neutrosophic Sets and Systems, Vol. 38, 2020.

[16] F. Smarandache, Introduction to Neutrosophic Statistics, USA: Sitech \& Education Publishing, 2014.

[17] Abobala, M., "Partial Foundation of Neutrosophic Number Theory", Neutrosophic Sets and Systems, Vol. 39 , 2021.

[18] Abobala, M., " Semi Homomorphisms and Algebraic Relations Between Strong Refined Neutrosophic Modules and Strong Neutrosophic Modules", Neutrosophic Sets and Systems, Vol. 39, 2021.

[19] T.Chalapathi and L. Madhavi,. "Neutrosophic Boolean Rings", Neutrosophic Sets and Systems, Vol. 33, pp. 5766, 2020.

[20] S. A. Edalatpanah., " Systems of Neutrosophic Linear Equations", Neutrosophic Sets and Systems, Vol. 33, pp. 92-104, 2020.

[21] Abobala, M., "On Some Algebraic Properties of n-Refined Neutrosophic Elements and n-Refined Neutrosophic Linear Equations", Mathematical Problems in Engineering, Hindawi, 2021

[22] Abobala, M., "A Study Of Nil Ideals and Kothe's Conjecture In Neutrosophic Rings", International Journal of Mathematics and Mathematical Sciences, hindawi, 2021

[23] Abobala, M., "Neutrosophic Real Inner Product Spaces", Neutrosophic Sets and Systems, Vol. 43, 2021.

[24] Abobala, M., "On Some Special Elements In Neutrosophic Rings and Refined Neutrosophic Rings", Journal of New Theory, vol. 33, 2020..

[25] Abobala, M., and Hatip, A., "An Algebraic Approch To Neutrosophic Euclidean Geometry", Neutrosophic Sets and Systems, Vol. 43, 2021.

[26] Abobala, M., " A Review On Recent Developments Of Neutrosophic Matrix Theory and Open Problems", Halarchives, 2021. .

[27] Abobala, M., Hatip, A., Olgun, N., Broumi, S., Salama, A,A., and Khaled, E, H., The algebraic creativity In The Neutrosophic Square Matrices, Neutrosophic Sets and Systems, Vol. 40, pp. 1-11, 2021.

[28] Chellamani, P., and Ajay, D., "Pythagorean neutrosophic Fuzzy Graphs", International Journal of Neutrosophic Science, Vol. 11, 2021.

DOI: https://doi.org/10.54216/IJNS.160202

Received: July 15, 2021 Accepted: November 09, 2021 
[29] Milles, S, Barakat, M, and Latrech, A., " Completeness and Compactness In Standard Single Valued neutrosophic Metric Spaces", International Journal of Neutrosophic Science, Vol.12, 2021.

[30] Es, Haydar, A., "A Note On neutrosophic Soft Menger Topological Spaces", International Journal of Neutrosophic Science, Vol.7, 2020.

[31] Ibrahim, M., and Abobala, M., "An Introduction To Refined Neutrosophic Number Theory", Neutrosophic Sets and Systems, Vol. 45, 2021.

[32] Ali, Rozina., " Neutrosophic Matrices and Their Properties", researchgat.net, 2021.

[33] Abobala, M, "n-Cyclic Refined Neutrosophic Algebraic Systems Of Sub-Indeterminacies, An Application To Rings and Modules", International Journal of Neutrosophic Science, Vol. 12, pp. 81-95 . 2020.

[34] Ali, R., "A Short Note On The Solution of n-Refined Neutrosophic Linear Diophantine Equations", International Journal Of Neutrosophic Science, Vol. 15, 2021.

DOI: https://doi.org/10.54216/IJNS.160202

Received: July 15, 2021 Accepted: November 09, 2021 